\title{
Systematic review of therapy used in relapsed or refractory diffuse large B-cell lymphoma and follicular lymphoma
}

\section{Future Science}

\author{
Aaron Galaznik*,1, Rachel Huelin², Michael Stokes ${ }^{3}$, Yelan Guo4, Meredith Hoog ${ }^{5}$, Tarun \\ Bhagnani ${ }^{6}$, Jill Bell ${ }^{1}$ \& Yaping Shou ${ }^{1}$ \\ ${ }^{1}$ Millennium Pharmaceuticals, Inc., a wholly owned subsidiary of Takeda Pharmaceutical Company Limited, 40 Landsdowne Street, \\ Cambridge, MA 02139, USA \\ ${ }^{2}$ Meta Research, Evidera, 500 Totten Pond Road, Fifth Floor, Waltham, MA 02451, USA \\ ${ }^{3}$ Data Analytics, Evidera, 7575 Trans-Canada Highway, Suite 404, St-Laurent, Quebec H4T 1V6, Canada \\ ${ }^{4}$ Meta Research, Evidera, Metro Building, $6^{\text {th }}$ Floor, 1 Butterwick, London W6 8DL, United Kingdom \\ ${ }^{5}$ Modelling and Simulation, Evidera, 7101 Wisconsin Avenue, Suite 1400, Bethesda, MD 20814, USA \\ ${ }^{6}$ Data Analytics, Evidera, 500 Totten Pond Road, Fifth Floor, Waltham, MA 02451, USA \\ *Author for correspondence: Tel.: +1 617444 4404; aaron.galaznik@takeda.com
}

To identify real-world evidence on outcomes from therapies for relapsed/refractory diffuse large B-cell lymphoma (DLBCL) and follicular lymphoma (FL), we systematically reviewed literature in Medline/Embase for DLBCL/FL-related articles on real-world results published during January 2012-May 2016. Among 33 included articles, therapies included stem cell transplant (SCT) and chemotherapy, including experimental regimens. The highest overall survival rates were observed for $\mathrm{SCT}$, long considered an optimal strategy following initial relapse. Prognoses were inferior among DLBCL patients receiving rituximab-based regimens rather than $\mathrm{SCT}$, particularly among studies that exclusively focused on those ineligible for SCT due to age or co-morbidity. A lack of viable treatment options for DLBCL/FL patients ineligible for SCT after relapse remains a significant gap in care.

Lay abstract: Non-Hodgkin lymphoma is the most prevalent blood cancer. Diffuse large B-cell lymphoma and follicular lymphoma account for nearly two-thirds of all non-Hodgkin lymphomas. Onethird of patients with diffuse large B-cell lymphoma continue on to relapsed or refractory disease. While follicular lymphoma tends to be less aggressive, relapses do occur. Stem cell transplant and chemotherapy/immunotherapy are the current treatment options for relapsed or refractory patients. However, many patients are ineligible for stem cell transplant, due to age or preexisting medical conditions, so safe and effective treatment choices for these patients are a must. The lack of viable treatment options highlights this unmet need.

First draft submitted: 8 February 2018; Accepted for publication: 23 May 2018; Published online: 19 July 2018

Keywords: chemotherapy $\bullet \mathrm{DLBCL} \bullet \mathrm{FL} \bullet$ overall survival $\bullet$ refractory $\bullet$ relapse $\bullet$ response $\bullet$ rituximab $\bullet$ stem cell therapy • systematic literature review

Non-Hodgkin lymphoma (NHL) is the most prevalent hematological malignancy and consists of a heterogeneous group of lymphoproliferative malignant diseases, characterized by a high degree of variety in pathology and clinical features. It is estimated that $85-90 \%$ of NHLs originate from B cells, and the remaining NHLs have a T-cell and natural killer cell lineage [1]. Diffuse large B-cell lymphoma (DLBCL) and follicular lymphoma (FL), both derived from B cells, are the two most common types of NHL, accounting for approximately $65 \%$ of all NHLs [1].

DLBCL is the most common of the NHLs and it is estimated to account for up to $40 \%$ of all NHLs [2]. Incidence of DLBCL increases with age and outcomes are variable. The National Comprehensive Cancer Network (NCCN) International Prognostic Index (IPI) is a scoring system that is used to assign patients to one of four different risk groups at the time of diagnosis - low, low-intermediate, high-intermediate and high [3,4]. This scoring system includes five predictors: age, lactate dehydrogenase levels, sites of involvement, Ann Arbor stage and Eastern 
Cooperative Oncology Group Performance Status. Based on the NCCN-IPI, 5-year overall survival (OS) rates range from $96 \%$ in low-risk patients to $33 \%$ in high-risk patients [3].

DLBCL exhibits a high degree of genetic, clinical and histologic variability; however, this observation has not been translated into current treatment algorithms [2,3]. The current treatment for DLBCL is R-CHOP - rituximab (R), a $\mathrm{CD}$-20-directed monoclonal antibody, given in combination with $\mathrm{CHOP}$, the standard chemotherapeutic regimen of cyclophosphamide, doxorubicin, vincristine and prednisone [3]. The number and timing of R-CHOP cycles may differ depending on whether the disease is localized or advanced. The addition of rituximab to the standard CHOP treatment regimen has had a significant effect on survival outcomes, improving them by approximately $15 \%$ [2]. Despite an overall good prognosis for patients diagnosed with DLBCL, approximately a third of patients will develop relapsed or refractory $(\mathrm{R} / \mathrm{R})$ disease. Treatments for R/R DLBCL patients include autologous stem cell transplant (auto-SCT) and chemotherapy/immunotherapy; however, there is limited clinical evidence on the real-world outcomes of these treatments. For patients who are candidates for auto-SCT and have chemosensitive disease, salvage chemotherapy followed by high-dose chemotherapy with auto-SCT is recommended. This has been shown to be effective in the long-term control of the disease in approximately half of these patients [2]. For patients who are not auto-SCT candidates due to age or co-morbidities, treatment with other chemotherapeutic agents with or without rituximab is recommended; however, prognosis for these patients is poor. Patients with progressive disease after three successive treatment regimens also have a poor prognosis, and are unlikely to derive any further benefit from currently available therapies $[2,3]$.

FL is the most common of the indolent NHLs and accounts for approximately $22 \%$ of all NHLs [3]. Most patients are approximately 50 years of age at diagnosis, at which point the disease is often already widespread [5]. Median survival ranges from 8 to 15 years with 5 -year survival outcomes ranging from 52.5 to $91 \%$. Three prognostic groups have been defined by the Follicular Lymphoma International Prognostic Index, a prognostic scoring system that is based on age, Ann Arbor stage, number of nodal sites involved, hemoglobin levels and serum lactate dehydrogenase levels [2]. Guidelines specifically for treating FL are based on Grade, and mainly apply to patients with Grade 1 or Grade 2 disease. Currently, the distinction between Grades 3A and 3B has not been shown to be clinically significant; FL Grade 3A may be treated as FL by some clinicians and as DLBCL by others [3]. However, FL Grade $3 \mathrm{~B}$ is commonly treated as DLBCL. Initial treatment for FL includes involved-site radiotherapy. An alternative to involved-site radiotherapy is immunotherapy, with or without chemotherapy, with or without radiotherapy [3]. For patients who relapse or who have progressive disease, hematopoietic SCT (autologous and allogeneic) can lead to an increase in progression-free survival (PFS) and OS $[1,3]$. Histological transformation of FL to DLBCL is generally associated with a poor clinical outcome, with a median OS of less than 2 years [2].

Since the addition of rituximab to the $\mathrm{CHOP}$ regimen approximately 15 years ago, there have been no additional approvals for any novel front-line therapies for either DLBCL or FL, nor any meaningful improvements to the R-CHOP survival rates [6]. With approximately a third of DLBCL patients experiencing R/R disease, many of whom are ineligible for SCT, there exists an unmet need, and the development of more effective therapies for these patients remains an important objective. Overall, while much has been published about NHL in general, there is a lack of clinical evidence specific to patients with R/R DLBCL or R/R FL. Furthermore, there is limited knowledge regarding healthcare utilization and the economic burden of these two diseases. Therefore, this review was designed to systematically collect and review information to evaluate the real-world evidentiary base for outcomes associated with therapies used to treat R/R DLBCL or R/R FL.

\section{Methods}

This systematic literature review was conducted according to the Preferred Reporting Items for Systematic Reviews and Meta-Analyses (PRISMA) guidelines. Using a prospectively established protocol, a systematic search was conducted in MEDLINE (via PubMed) and Embase to identify studies that report real-world treatment outcomes in patients with DLBCL and FL published from 1 January 2012 to 11 May 2016. We also reviewed conference abstracts for the two most recent meetings (as of June 2016) of the following groups: American Society of Clinical Oncology, European Society for Medical Oncology, American Society of Hematology, European Hematology Association and International Society for Pharmacoeconomics and Outcomes Research.

In the first round of screening, all titles and abstracts were screened by a single investigator against the inclusion and exclusion criteria, using the PICOS-T elements (participants, interventions, comparisons, outcomes, study design and time period; Table 1). Although the focus of this manuscript is R/R DLBCL/FL, the patient population queried at this stage was adult patients with DLBCL or FL. A senior investigator validated $10 \%$ of the rejected 
Table 1. Study selection criteria (participants, interventions, comparisons, outcomes, study design and time period).

\begin{tabular}{|c|c|}
\hline Criteria & Inclusion criteria \\
\hline Population(s) & Adult patients ( $\geq 18$ years) with diffuse large B-cell lymphoma or follicular lymphoma \\
\hline Interventions & Chemotherapeutic/immunotherapy agents licensed and under investigation for these conditions, where applicable \\
\hline Comparisons & Not applicable \\
\hline Outcomes & $\begin{array}{l}\text { - Effectiveness: treatment response rates (cytogenetic and hematologic responses), duration of response, progression-free } \\
\text { survival, overall survival } \\
\text { - Tolerability/safety: overall/severe AEs, withdrawals, Grade } 3 \text { to } 4 \text { AEs } \\
\text { - Economic: ICERs, QALYs, life-years gained, costs and healthcare resource use } \\
\text { - HRQoL/patient-reported outcomes/utilities: patient-reported outcomes, HRQoL outcomes (measured via generic [e.g., EQ-5D] } \\
\text { or disease-specific instruments [e.g., EORTC QLQ-C30]) } \\
\text { - Treatment patterns and guidelines }\end{array}$ \\
\hline Time & $\begin{array}{l}\text { Indexed database: } 1 \text { January } 2012 \text { to } 10 \text { May } 2016 \\
\text { Gray literature: Two most recent meetings }\end{array}$ \\
\hline Study design & $\begin{array}{l}\text { - Clinical effectiveness and safety, treatment patterns: observational studies } \\
\text { - Economic evaluations: economic analyses (e.g., CEMs, CBAs, CUAs, CMAs) } \\
\text { - HRQoL/patient-reported outcomes/utilities: all study designs } \\
\text { - Costs and resource use: all study designs } \\
\text { - Clinical guidelines }\end{array}$ \\
\hline Other & $\begin{array}{l}\text { English language only; } \\
\text { Geographic emphasis on the USA, France, Germany, Italy, Spain, United Kingdom and Japan }\end{array}$ \\
\hline \multicolumn{2}{|c|}{$\begin{array}{l}\text { AE: Adverse event; CBA: Cost-benefit analysis; CEM: Cost-effectiveness model; CMA: Comparative market analysis; CUA: Cost-utility analysis; EORTC QLQ-C30: Eu- } \\
\text { ropean Organisation for Research and Treatment of Cancer Quality of Life Questionnaire-C30; EQ-5D: EuroQol five dimensions questionnaire; HRQoL: Health-related } \\
\text { quality of life; ICER: Incremental cost-effectiveness ratio; QALY: Quality-adjusted life-year. }\end{array}$} \\
\hline
\end{tabular}

abstracts to confirm accuracy. No study was excluded at the abstract level based solely on insufficient information. Full-text articles for the accepted abstracts were retrieved for in-depth review in the second round of screening, conducted by a single investigator using the same inclusion and exclusion criteria applied at the abstract level. Only studies enrolling $\geq 50$ patients were included, and a geographic emphasis was placed on studies conducted primarily in France, Germany, Italy, Spain, the United Kingdom, the USA and Japan. A second investigator confirmed all excluded studies; any discrepancies were resolved by a third investigator. Throughout the process, discrepancies were addressed by consensus and with input from a third investigator if necessary.

The literature search included publications (English only) on randomized controlled trials (RCTs), observational studies and economic evaluations that reported clinical effectiveness, tolerability/safety, economic or health-related quality of life/patient reported outcomes in adult patients with DLBCL or FL.

Full data extraction was performed on all studies included following the second round of screening. Extracted data included study descriptors; patient characteristics; treatment-level information; outcomes (efficacy, safety/tolerability, economic, health-related quality of life and patient reported outcomes); treatment patterns and clinical guidelines.

\section{Results}

At the abstract level, a total of 2599 unique citations were identified. After these abstracts were screened, 255 had a sample size of $\geq 50$ patients and were selected for full-text review; 2344 were excluded. The main reasons for exclusion during the full-text review were the patient population not meeting selection criteria (e.g., no R/R DLBCL or R/R FL patients included or mixed populations), the article not reporting data on a treatment-related effectiveness outcome $(n=37)$ or the study enrolling patients outside of the review's geographical scope $(n=39)$. After applying all criteria, 33 articles [7-39] examining either efficacy or effectiveness of therapies administered to patients with R/R DLBCL or R/R FL were identified. The PRISMA study attrition diagram is shown in Figure 1.

Of the 33 included studies, 21 were full-text papers and 12 were scientific conference abstracts. There were 18 articles reporting on patients with R/R DLBCL and 15 reporting on R/R FL. The majority of publications had observational study designs $(n=21)$.

\section{Summary of studies by treatment \& patient characteristics}

Of the 18 studies evaluating patients with R/R DLBCL, SCT was the most common treatment, examined in seven articles. Six studies looked at rituximab-based chemo, three at experimental chemo and two discussed carmustine 


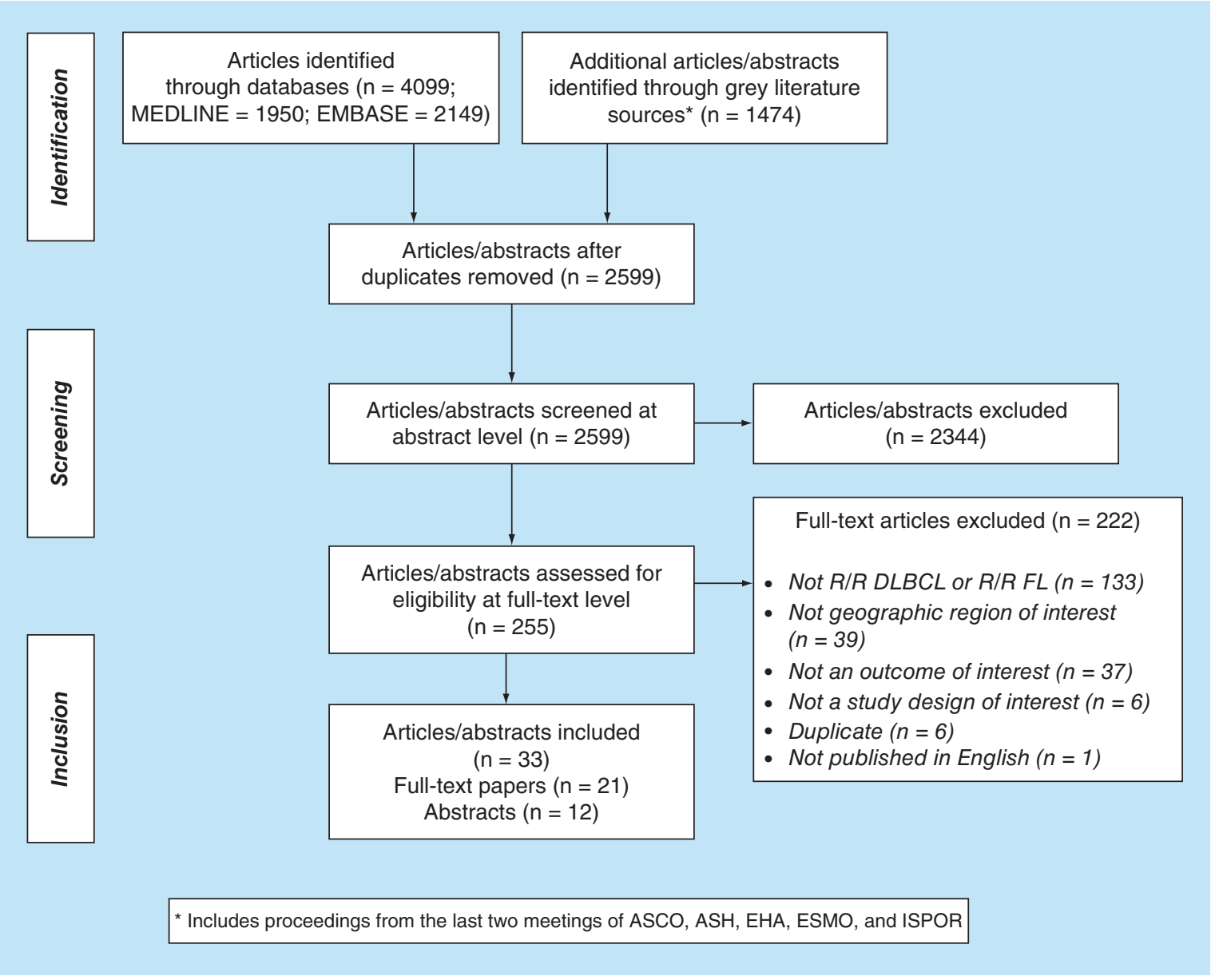

Figure 1. The Preferred Reporting Items for Systematic Reviews and Meta-Analyses study attrition diagram. ASCO: American Society of Clinical Oncology; ASH: American Society of Hematology; DLBCL: Diffuse large B cell lymphoma; EHA: European Hematology Association; ESMO: European Society for Medical Oncology; FL: Follicular lymphoma; ISPOR: International Society for Pharmacoeconomics and Outcomes Research.

(BCNU), etoposide, Ara-C and melphalan (BEAM) conditioning. Experimental chemotherapy was defined as any drug regimen that did not include rituximab, or that was investigated as part of a clinical trial. Most publications reported data on disease stage, and enrolled patients across all stages. Proportions of patients with Stage 3 or 4 DLBCL ranged from 31 to $76 \%$. In studies of R/R FL, the majority of articles examined SCT $(n=9)$, followed by experimental chemotherapy $(\mathrm{n}=3)$, Yttrium-90 Ibritumomab Tiuxetan (90Y-IT; $\mathrm{n}=1)$, watchful waiting $(\mathrm{n}=1)$ and salvage treatment $(\mathrm{n}=1)$.

\section{Clinical outcomes of treatment for R/R DLBCL Observational study designs}

Twelve of the studies examining R/R DLBCL were of an observational study design (Table 2). Of these, the majority (7/12) investigated the effectiveness of either autologous (auto-) SCT or allogeneic (allo-) SCT, and reported on OS; OS ranged from $18 \%$ at 3 years for the subset of patients with non-germinal center B-cell-like DLBCL, to $64 \%$ at 4 years in an overall population of R/R DLBCL $[8,11,14,15,20,21,26]$. One study [21] directly compared auto-SCT with allo-SCT and found that myeloablative conditioning used prior to allo-SCT was significantly inferior to auto-SCT for both OS (hazard ratio [HR]: 1.9; 95\% CI: 1.5-2.4) and PFS (HR: 1.4; 95\% CI: 1.1-1.7) outcomes, after adjusting for baseline and demographic characteristics. However, for reduced-intensity conditioning (RIC) alloSCT, the OS disadvantage (HR: 1.3; 95\% CI: 1.0-1.8) was of only borderline significance, and the disadvantage for PFS was not significant (HR: 1.2; 95\% CI: 0.9-1.6). Another study, using data from the European Group for Blood Marrow Transplantation database, compared unrelated donor allo-SCT with allografts from sibling hematopoietic 
SCT [8]. At 3 years, neither OS ( 42 vs 37\% for sibling and unrelated donors, respectively) nor PFS (35 vs 35\% for sibling and unrelated donors, respectively) was associated with donor type. This was confirmed in multivariate analyses. Among all of the studies investigating SCT, the highest OS rate of $71 \%$ was reported in a study examining auto-SCT at a median follow-up of 39 months [9]. This study also found that patients who relapsed as a result of chemosensitivity had higher 3-year event-free survival (EFS; 66\%) compared with refractory patients (35\%, $\mathrm{p}=0.001)$.

The remainder of the observational studies reported on outcomes in R/R DLBCL patients treated with either rituximab-based regimens $(n=3)$ or BEAM conditioning $(n=1)$. The studies investigating rituximab-based regimens reported median OS ranging from 10.8 to 21.0 months [7,10]. Farooq et al. showed that patients in complete response at the end of first-line therapy have a significant survival advantage relative to those with stable disease or partial response (median OS: 21 vs 6.8 months, respectively) [10]. A study examining longer-term survival of 5 years from progression found that primary chemorefractory patients had lower OS (15\%) compared with relapsed patients (42\%) [24]. In the one observational study evaluating the effectiveness of BEAM conditioning regimens [15], 5-year OS rates showed a slight advantage for Z-BEAM over BEAM/R (86 vs 69\%) during the 2007-2010 period. However, no difference in the rate of 5-year disease-free survival (DFS) was observed.

There were limited data on response outcomes. Response rates were reported in only three of the observational studies identified. Overall response rates (ORRs) of $49 \%$ were reported in a study examining allo-SCT after prior auto-SCT; this was the only SCT study reporting data on response outcomes [20]. In another study, an ORR of 50\% and complete response of $28 \%$ were reported for R/R DLBCL patients treated with rituximab + bendamustine [7]. The article by Rovira et al. [24] also compared complete response outcomes in primary chemorefractory and relapsed patients. As with OS, the authors demonstrated a significant advantage with respect to complete response for relapsed patients (59 vs 17\% for relapse vs chemorefractory during the $2002-2012$ period).

\section{Clinical trials}

Six publications discuss trials for R/R DLBCL treatments (Table 3). Three clinical trials examined the efficacy of experimental chemotherapy regimens. Czuczman et al. compared lenalidomide with investigator's choice regimen (gemcitabine, rituximab, etoposide or oxaliplatin) and found that median OS (31 vs 24.6 weeks), median EFS (13.6 vs 7.9 weeks) and the ORR (28 vs 11.8\%) were all improved with lenalidomide compared with investigator's choice [27]. The other two articles were single-arm studies examining the vorinostat + gemcitabine + busulfan/melphalan regimen [18] and fostamatin [12]. Nieto et al. concluded that the vorinostat regimen induced high complete response rates (73\%) and delivered promising early outcomes in R/R DLBCL patients; $73 \%$ of the study population was alive at a median follow-up of 25 months [18]. The study examining fostamatinib found that, although it was generally well tolerated at the 200-mg dose, efficacy was limited, with only $3 \%$ of patients achieving any type of response [12].

The results of a Phase II clinical trial of rituximab plus vinorelbine, ifosfamide, mitoxantrone and prednisone (RNIMP) suggested this regimen as a promising option in relapsed DLBCL [13]. The highest OS (median 28.4 months) and PFS (median 10.5 months) outcomes for rituximab-based regimens were reported in this study. Another Phase II study evaluating the BEAM conditioning regimen had the highest rates of complete response (84\%) [28] out of all the clinical trials and observational studies in R/R DLBCL included. In a Phase II study of bendamustine plus rituximab (BR) in patients with R/R DLBCL who were not candidates for SCT, an ORR of 63\% and a CR rate of $37 \%$ was observed, indicating that $\mathrm{BR}$ is a promising salvage regimen in DLBCL patients with limited therapeutic options [19].

\section{Clinical outcomes of treatment for R/R FL}

Observational study designs

Similar to R/R DLBCL, the majority of the ten observational studies in R/R FL (8/10) focused on either the auto-SCT or allo-SCT treatment settings (Table 4). The majority of studies reported 5-year OS rates from 51 to $72 \%[16,17,21,23,30,33,37]$. This is unsurprising as SCT is commonly regarded as the only curative option in FL. One study found that strong evidence of response based on negative positron emission tomography-computed tomography findings prior to allo-SCT was a strong prognostic factor in patients with relapsing chemosensitive FL [29], with 3-year OS rates of $90 \%$ observed in this subset of patients.

Several studies compared the outcomes of patients treated with allo-SCT versus auto-SCT with conflicting results, depending upon the subgroup of FL patients included and/or the time period of evaluation [16,17,21]. Klyuchnikov 
Table 2. Summary of observational studies examining clinical outcomes of treatment for relapsed or refractory diffuse large B-cell lymphoma.

\begin{tabular}{|c|c|c|c|c|c|c|c|c|}
\hline $\begin{array}{l}\text { Country or } \\
\text { region }\end{array}$ & Design & $\begin{array}{l}\text { Treatment } \\
\text { setting }\end{array}$ & Sample size & $\begin{array}{l}\text { Follow-up } \\
\text { (months) }\end{array}$ & Survival/progression & Response & $\begin{array}{l}\text { Study (year), } \\
\text { Ref. }\end{array}$ & Source \\
\hline $\begin{array}{l}\text { France, } \\
\text { Germany, Italy, } \\
\text { Spain, UK }\end{array}$ & $\begin{array}{l}\text { Retrospective } \\
\text { cohort }\end{array}$ & $\begin{array}{l}\text { Auto-SCT vs } \\
\text { Allo-SCT }\end{array}$ & 6717 & $\begin{array}{l}\text { Median } 18 \text { mos } \\
\text { (among } \\
\text { survivors) }\end{array}$ & $\begin{array}{l}\text { OS at } 4 \text { yrs: } \\
\text { Auto-SCT: } 54 \% \\
\text { MAC-allo-SCT: } 30 \% \\
\text { RIC-allo-SCT: } 38 \% \\
\text { PFS at } 4 \text { yrs: } \\
\text { Auto-SCT: } 43 \% \\
\text { MAC-allo-SCT: } 28 \% \\
\text { RIC-allo-SCT: } 28 \%\end{array}$ & NR & $\begin{array}{l}\text { Robinson } \\
\text { et al. } \\
(2016),[21]\end{array}$ & Full text \\
\hline $\begin{array}{l}\text { France, } \\
\text { Germany, Italy, } \\
\text { Spain, UK }\end{array}$ & $\begin{array}{l}\text { Retrospective } \\
\text { cohort }\end{array}$ & $\begin{array}{l}\text { Allo-SCT: } \\
\text { unrelated donor } \\
\text { vs sib-SCT }\end{array}$ & 473 & $\begin{array}{l}\text { Median } 45 \text { mos } \\
\text { (among } \\
\text { survivors) }\end{array}$ & $\begin{array}{l}\text { OS at } 3 \text { yrs: } \\
\text { Sibling: } 42 \% \\
\text { Unrelated: } 37 \% \\
\text { PFS at } 3 \text { yrs: } \\
\text { Sibling: } 35 \% \\
\text { Unrelated: } 35 \%\end{array}$ & NR & $\begin{array}{l}\text { Avivi et al. } \\
\text { (2014), [8] }\end{array}$ & Full text \\
\hline $\begin{array}{l}\text { France, } \\
\text { Germany, Italy, } \\
\text { Spain, UK }\end{array}$ & $\begin{array}{l}\text { Retrospective } \\
\text { cohort }\end{array}$ & Auto-SCT & 86 & Median 39 mos & $\begin{array}{l}\text { OS: } 71 \% \text { at median } \\
\text { follow-up of } \\
39 \text { months } \\
\text { EFS at } 3 \text { yrs: } \\
\text { Chemosensitive: } \\
66 \% \\
\text { Refractory: } 35 \% \\
\text { Transplant in } \\
\text { complete or partial } \\
\text { response: } 100 \%\end{array}$ & NR & $\begin{array}{l}\text { Avivi et al. } \\
\text { (2014), [9] }\end{array}$ & Abstract \\
\hline USA & Observational & $\begin{array}{l}\text { Allo-SCT after } \\
\text { prior auto-SCT }\end{array}$ & 503 & $\begin{array}{l}\text { Median } 55 \text { mos } \\
\text { (among } \\
\text { survivors) }\end{array}$ & $\begin{array}{l}\text { OS at } 3 \text { yrs: } \\
\text { Overall: } 37 \% \\
\text { Low risk: } 43 \% \\
\text { Intermediate risk: } \\
25 \% \\
\text { High risk: } 14 \% \\
\text { PFS at } 3 \text { yrs: } \\
\text { Overall: } 31 \% \text {; } \\
\text { Low risk: } 38 \% \\
\text { Intermediate risk: } \\
\text { 19\% } \\
\text { High risk: } 10 \%\end{array}$ & NR & $\begin{array}{l}\text { Fenske et al. } \\
\text { (2015), [11] }\end{array}$ & Full text \\
\hline Italy & $\begin{array}{l}\text { Retrospective } \\
\text { cohort }\end{array}$ & $\begin{array}{l}\text { Allo-SCT after } \\
\text { prior auto-SCT }\end{array}$ & 165 & $\begin{array}{l}\text { Median } 39 \text { mos } \\
\text { (among } \\
\text { survivors) }\end{array}$ & $\begin{array}{l}\text { OS } \\
1 \text { yr: } 55 \% \\
3 \text { yrs: } 42 \% \\
5 \text { yrs: } 39 \% \\
\text { PFS } \\
1 \text { yr: } 48 \% \\
3 \text { yrs: } 34 \% \\
5 \text { yrs: } 31 \%\end{array}$ & $\begin{array}{l}\text { ORR: } 49 \% \\
\text { CR: } 65 \% \text { (among } \\
\text { evaluable patients) }\end{array}$ & $\begin{array}{l}\text { Rigacci et al. } \\
\text { (2012), [20] }\end{array}$ & Full text \\
\hline USA & $\begin{array}{l}\text { Retrospective } \\
\text { cohort }\end{array}$ & $\begin{array}{l}\text { Allo-SCT in } \\
\text { patients with } \\
\text { relapse/ } \\
\text { progression } \\
\text { after prior } \\
\text { chemo }\end{array}$ & $\begin{array}{l}\text { Overall: } 101 \\
\text { PMBL: } 17 \\
\text { GBC: } 62 \\
\text { Non-GBC: } 22\end{array}$ & $\begin{array}{l}\text { Median mos } \\
\text { PMBL: } 52 \\
\text { GBC: } 63 \\
\text { Non-GBC: } 29\end{array}$ & $\begin{array}{l}\text { OS at } 3 \text { yrs: } \\
\text { PMBL: } 46 \% \\
\text { GBC: } 52 \% \\
\text { Non-GBC: } 18 \% \\
\text { PFS at } 3 \text { yrs: } \\
\text { PMBL: } 41 \% \\
\text { GBC: } 39 \% \\
\text { Non-GBC: } 12 \%\end{array}$ & NR & $\begin{array}{l}\text { Khouri et al. } \\
\text { (2014), [26] }\end{array}$ & Abstract \\
\hline
\end{tabular}

Auto/allo-SCT: Autologous/allogeneic stem cell transplantation; BEAM: Etoposide, Ara-C and melphalan; CHOP: Cyclophosphamide, doxorubicin, vincristine and prednisone; CR: Complete response; CTD: Circulating tumor DNA; DFS: Disease-free survival; DOR: Duration of response; EFS: Event-free survival; GBC: Germinal center B-cell-like; MAC: Myeloablative conditioning; mos: Months; NR: Not reported; ORR: Overall response rate; OS: Overall survival; PFS: Progression-free survival; PMBL: Primary mediastinal B-cell lymphoma; PR: Partial response; R-DHAP: Rituximab dexamethasone, cytarabine and cisplatin; RIC: Reduced-intensity conditioning; R/R: Relapsed/refractory; SCT: Stem cell transplant; sib-SCT: Sibling stem cell transplantation; yrs: Years. 
Table 2. Summary of observational studies examining clinical outcomes of treatment for relapsed or refractory diffuse large B-cell lymphoma (cont.).

\begin{tabular}{|c|c|c|c|c|c|c|c|c|}
\hline $\begin{array}{l}\text { Country or } \\
\text { region }\end{array}$ & Design & $\begin{array}{l}\text { Treatment } \\
\text { setting }\end{array}$ & Sample size & $\begin{array}{l}\text { Follow-up } \\
\text { (months) }\end{array}$ & Survival/progression & Response & $\begin{array}{l}\text { Study (year), } \\
\text { Ref. }\end{array}$ & Source \\
\hline USA & $\begin{array}{l}\text { Retrospective } \\
\text { cohort }\end{array}$ & $\begin{array}{l}\text { Allo-SCT in } \\
\text { patients with } \\
\text { relapse/ } \\
\text { progression } \\
\text { after prior } \\
\text { chemo }\end{array}$ & 53 & Median 56 mos & $\begin{array}{l}\text { OS at } 4 \text { yrs: } \\
\text { Overall: } 64 \% \\
\text { CTD positive: } 38 \% \\
\text { CTD negative: } 67 \% \\
\text { PFS at } 4 \text { yrs: } \\
\text { Overall: } 46 \% \\
\text { CTD positive: } 13 \% \\
\text { CTD negative: } 48 \%\end{array}$ & NR & $\begin{array}{l}\text { Herrera et al. } \\
\text { (2015), [14] }\end{array}$ & Abstract \\
\hline Italy & $\begin{array}{l}\text { Retrospective } \\
\text { cohort }\end{array}$ & $\begin{array}{l}\text { Rituximab-based } \\
\text { regimens: } \\
\text { rituximab + ben- } \\
\text { damustine }\end{array}$ & 55 & $\begin{array}{l}\text { Median } 10.6 \\
\text { mos }\end{array}$ & $\begin{array}{l}\text { Median OS: } 10.8 \\
\text { mos } \\
\text { Median PFS: } 8.8 \\
\text { mos }\end{array}$ & $\begin{array}{l}\text { ORR: } 50 \% \\
\text { CR: } 28 \% \\
\text { DOR: } 20.3 \text { mos } \\
\text { (among patients } \\
\text { with PR or CR) }\end{array}$ & $\begin{array}{l}\text { Arcari et al. } \\
\text { (2015), [7] }\end{array}$ & Full text \\
\hline USA & $\begin{array}{l}\text { Prospective } \\
\text { study }\end{array}$ & $\begin{array}{l}\text { Rituximab-based } \\
\text { regimens: ritux- } \\
\text { imab + CHOP }\end{array}$ & 258 & Median 59 yrs & $\begin{array}{l}\text { Median OS } \\
\text { Responsive disease } \\
\text { (CR or PR): } 21.0 \text { mos } \\
\text { Stable or } \\
\text { progressive disease: } \\
6.8 \text { mos } \\
\text { PFS at } 2 \text { yrs: } 45 \% \\
\text { (post-transplant) }\end{array}$ & NR & $\begin{array}{l}\text { Farooq et al. } \\
\text { (2015), [10] }\end{array}$ & Abstract \\
\hline $\begin{array}{l}\text { France, } \\
\text { Germany, Italy, } \\
\text { Spain, UK }\end{array}$ & $\begin{array}{l}\text { Retrospective } \\
\text { cohort }\end{array}$ & $\begin{array}{l}\text { Rituximab-based } \\
\text { regimens: } \\
\text { Rituximab } \\
\text { added as } \\
\text { salvage therapy }\end{array}$ & 144 & $\begin{array}{l}\text { Median } 6.5 \text { yrs } \\
\text { (among } \\
\text { survivors) }\end{array}$ & $\begin{array}{l}\text { OS, median from } \\
\text { relapse was } 1.12 \text { yrs } \\
\text { for R/R CR patients } \\
\text { 5-yr survival from } \\
\text { progression: } \\
\text { Primary } \\
\text { chemorefractory } \\
\text { (1991-2001): } 6 \% \\
\text { Primary } \\
\text { chemorefractory } \\
\text { (2002-2012): } 15 \% \text {; } \\
\text { Relapsed } \\
\text { (1991-2001): } 33 \% \\
\text { Relapsed } \\
\text { (2002-2012): } 42 \%\end{array}$ & $\begin{array}{l}\text { CR: } \\
\text { Primary } \\
\text { chemorefractory } \\
\text { (1991-2001): } 3 \% \\
\text { Primary } \\
\text { chemorefractory } \\
\text { (2002-2012): } 17 \% \\
\text { Relapsed } \\
\text { (1991-2001): 51\% } \\
\text { Relapsed } \\
\text { (2002-2012): 59\% } \\
\text { PR: } \\
\text { Primary } \\
\text { chemorefractory } \\
\text { (1991-2001): } 2 \% \\
\text { Primary } \\
\text { chemorefractory } \\
\text { (2002-2012): } 26 \% \\
\text { Relapsed } \\
\text { (1991-2001): 39\% } \\
\text { Relapsed } \\
\text { (2002-2012): } 26 \%\end{array}$ & $\begin{array}{l}\text { Rovira et al. } \\
\text { (2015), [24] }\end{array}$ & Full text \\
\hline USA & $\begin{array}{l}\text { Retrospective } \\
\text { cohort }\end{array}$ & $\begin{array}{l}\text { Beam } \\
\text { conditioning: } \\
\text { BEAM/R vs } \\
\text { Z-BEAM }\end{array}$ & $\begin{array}{l}\text { Overall: } 113 \\
\text { Group A: } 57 \\
\text { Group B: } 26 \\
\text { Group C: } 16 \\
\text { Group D: } 14\end{array}$ & $\begin{array}{l}\text { Median yrs } \\
\text { Group A: } 11.8 \\
\text { Group B: } 8.1 \\
\text { Group C: } 4.2 \\
\text { Group D: } 4.9\end{array}$ & $\begin{array}{l}\text { OS at } 5 \text { yrs } \\
\text { Group A: } 74 \% \\
\text { Group B: } 73 \% \\
\text { Group C: } 69 \% \\
\text { Group D: } 86 \% \\
\text { DFS at } 5 \text { yrs } \\
\text { Group A: } \\
62 \% \text { Group B: } 65 \% \\
\text { Group C: } 63 \% \\
\text { Group D: } 63 \%\end{array}$ & NR & $\begin{array}{l}\text { Khouri et al. } \\
\text { (2015), [15] }\end{array}$ & Abstract \\
\hline Germany & $\begin{array}{l}\text { Retrospective } \\
\text { cohort }\end{array}$ & $\begin{array}{l}\text { Modified } \\
\text { R-DHAP regimen }\end{array}$ & 122 & NR & NR & $\begin{array}{l}\text { ORR: } 74 \%, C R: 17 \% \text {, } \\
\text { PR: } 32 \%\end{array}$ & $\begin{array}{l}\text { Lisenko et al. } \\
\text { (2015), [25] }\end{array}$ & Abstract \\
\hline \multicolumn{9}{|c|}{$\begin{array}{l}\text { Auto/allo-SCT: Autologous/allogeneic stem cell transplantation; BEAM: Etoposide, Ara-C and melphalan; CHOP: Cyclophosphamide, doxorubicin, vincristine and prednisone; CR: } \\
\text { Complete response; CTD: Circulating tumor DNA; DFS: Disease-free survival; DOR: Duration of response; EFS: Event-free survival; GBC: Germinal center B-cell-like; MAC: Myeloablative } \\
\text { conditioning; mos: Months; NR: Not reported; ORR: Overall response rate; OS: Overall survival; PFS: Progression-free survival; PMBL: Primary mediastinal B-cell lymphoma; PR: Partial } \\
\text { response; R-DHAP: Rituximab dexamethasone, cytarabine and cisplatin; RIC: Reduced-intensity conditioning; R/R: Relapsed/refractory; SCT: Stem cell transplant; sib-SCT: Sibling stem } \\
\text { cell transplantation; yrs: Years. }\end{array}$} \\
\hline
\end{tabular}


Table 3. Summary of randomized controlled trial studies examining clinical outcomes of treatment for relapsed or refractory diffuse large B-cell lymphoma.

\begin{tabular}{|c|c|c|c|c|c|c|c|c|}
\hline Country/region & Design & $\begin{array}{l}\text { Treatment } \\
\text { setting }\end{array}$ & Sample size & $\begin{array}{l}\text { Follow-up } \\
\text { (months) }\end{array}$ & $\begin{array}{l}\text { Survival/ } \\
\text { progression }\end{array}$ & Response & $\begin{array}{l}\text { Study (year), } \\
\text { ref. }\end{array}$ & Source \\
\hline USA & $\begin{array}{l}\text { Single arm, } \\
\text { dose-finding } \\
\text { trial }\end{array}$ & $\begin{array}{l}\text { Experimental } \\
\text { chemo: vorinos- } \\
\text { tat + gem + busulf }\end{array}$ & 52 & Median 25 mos & $\begin{array}{l}\text { OS: } 73 \% \\
\text { EFS: } 61.5 \%\end{array}$ & $\begin{array}{l}\text { ORR: } 96 \% \text { (among } \\
\text { DLBCL and } \\
\text { measurable disease) } \\
\text { CR: } 73 \% \text { (among } \\
\text { DLBCL and } \\
\text { measurable disease) }\end{array}$ & $\begin{array}{l}\text { Nieto et al. } \\
\text { (2015), [18] }\end{array}$ & Full text \\
\hline UK, USA & Phase II trial & $\begin{array}{l}\text { Experimental } \\
\text { chemo: } \\
\text { fostamatinib }\end{array}$ & 68 & NR & NR & $\begin{array}{l}\text { ORR: } 3 \% \\
\text { Clinical benefit } \\
\text { (ORR + SD): } 13 \%\end{array}$ & $\begin{array}{l}\text { Flinn et al. } \\
\text { (2014), [12] }\end{array}$ & Abstract \\
\hline NR & $\begin{array}{l}\text { Phase II or Phase } \\
\text { III }\end{array}$ & $\begin{array}{l}\text { Experimental } \\
\text { chemo: } \\
\text { lenalidomide vs } \\
\text { investigator's } \\
\text { choice } \\
\text { (gemcitabine, } \\
\text { rituximab, } \\
\text { etoposide or } \\
\text { oxaliplatin) }\end{array}$ & 102 & NR & $\begin{array}{l}\text { Median OS (weeks) } \\
\text { Overall } \\
\text { LEN: } 31 \\
\text { IC: } 24.6 \\
\text { Immunochemistry } \\
\text { LEN GCB: } 30 \\
\text { IC GCB: } 24.9 \\
\text { LEN non-GCB: } 32.3 \\
\text { IC non-GCB: } 20.4 \\
\text { Gene expression } \\
\text { profiling } \\
\text { LEN GCB: } 30 \\
\text { IC GCB: } 20.1 \\
\text { LEN non-GCB: } 108.4 \\
\text { IC non-GCB: } 18.6 \\
\text { Median PFS (weeks) } \\
\text { Overall } \\
\text { LEN: } 13.6 \\
\text { IC: } 7.9 \\
\text { Immunochemistry } \\
\text { LEN GCB: } 10.1 \\
\text { IC GCB: } 9.0 \\
\text { LEN non-GCB: } 15.1 \\
\text { IC non-GCB: } 7.1 \\
\text { Gene expression } \\
\text { profiling } \\
\text { LEN GCB: } 13.2 \% \\
\text { IC GCB: } 7.1 \% \\
\text { LEN non-GCB: } \\
82.0 \% \\
\text { IC non-GCB: } 6.2 \%\end{array}$ & $\begin{array}{l}\text { ORR } \\
\text { Overall } \\
\text { LEN: } 27.5 \% \\
\text { IC: } 11.8 \% \\
\text { Immunochemistry } \\
\text { LEN GCB: } 26.1 \% \\
\text { IC GCB: } 12.0 \% \\
\text { LEN non-GCB: } \\
28.6 \% \\
\text { IC non-GCB: } 11.5 \% \\
\text { Gene expression } \\
\text { profiling } \\
\text { LEN GCB: } 21.4 \% \\
\text { IC GCB: } 12.5 \% \\
\text { LEN non-GCB: } \\
45.5 \% \\
\text { IC non-GCB: } 18.8 \%\end{array}$ & $\begin{array}{l}\text { Czuczman } \\
\text { et al. } \\
(2014),[27]\end{array}$ & Abstract \\
\hline France & Phase II trial & $\begin{array}{l}\text { Rituximab-based } \\
\text { regimens: ritux- } \\
\text { imab + NIMP }\end{array}$ & 50 & $\begin{array}{l}\text { Median } 51.1 \\
\text { mos (among } \\
\text { survivors) }\end{array}$ & $\begin{array}{l}\text { Median OS: } 28.4 \\
\text { mos } \\
\text { Median PFS: } 10.5 \\
\text { mos }\end{array}$ & $\begin{array}{l}\text { ORR: } 66 \% \text { (after } \\
\text { three cycles) } \\
\text { CR: } 30 \% \text { (after } \\
\text { three cycles) }\end{array}$ & $\begin{array}{l}\text { Gyan et al. } \\
\text { (2013), [13] }\end{array}$ & Full text \\
\hline Japan, Korea & Phase II study & $\begin{array}{l}\text { Rituximab-based } \\
\text { regimens: } \\
\text { rituximab + ben- } \\
\text { damustine }\end{array}$ & 59 & Median 4.7 mos & $\begin{array}{l}\text { Median PFS: } 6.7 \\
\text { mos }\end{array}$ & $\begin{array}{l}\text { ORR: } 62.7 \% \\
\text { CR: } 37.3 \%\end{array}$ & $\begin{array}{l}\text { Ohmachi } \\
\text { et al. } \\
\text { (2013), [19] }\end{array}$ & Full text \\
\hline $\begin{array}{l}\text { France, } \\
\text { Germany, Italy, } \\
\text { Spain, UK }\end{array}$ & Phase II trial & $\begin{array}{l}\text { Beam } \\
\text { conditioning: } \\
\text { conventional } \\
\text { BEAM }\end{array}$ & 57 & $\begin{array}{l}\text { Median } 10.5 \\
\text { mos }\end{array}$ & NR & $\begin{array}{l}\text { CR: } 84 \% \\
\text { At } 10.5 \text { months, } \\
65 \% \text { alive and in CR }\end{array}$ & $\begin{array}{l}\text { Isidori et al. } \\
\text { (2015), [28] }\end{array}$ & Abstract \\
\hline
\end{tabular}

et al. found that Grade 1/2 FL patients treated with auto-SCT had superior OS outcomes compared with RICallo-SCT (OS: 5-year 74 vs 66\%, p = 0.05) [16]. However, in higher-grade patients, no significant difference in OS was observed [17]. In another study by Robinson et al., median OS in the auto-SCT arm (59 months) was similar to RIC-allo-SCT (60 months) [23]. With respect to relapse/progression, significantly higher rates at 5 years were observed for allo-SCT vs auto-SCT across the three studies (Klyuchnikov et al. (2015) [PFS: 58 vs 41\%]; Klyuchnikov et al. (2016) [PFS: 51 vs 36\%] and Robinson et al. [PFS: 57 vs 48\%]) [16,17,23]. Finally, in another study comparing BEAM-auto-SCT with BEAM-allo-SCT in 171 patients with relapsed FL [33], OS and PFS were 


\begin{tabular}{|c|c|c|c|c|c|c|c|c|}
\hline Country/region & Design & $\begin{array}{l}\text { Treatment } \\
\text { setting }\end{array}$ & Sample size & $\begin{array}{l}\text { Follow-up } \\
\text { (months) }\end{array}$ & $\begin{array}{l}\text { Survival/ } \\
\text { progression }\end{array}$ & Response & $\begin{array}{l}\text { Study (year), } \\
\text { Ref. }\end{array}$ & Source \\
\hline $\begin{array}{l}\text { France, Germany, } \\
\text { Italy, Spain, UK }\end{array}$ & $\begin{array}{l}\text { Retrospective } \\
\text { cohort }\end{array}$ & $\begin{array}{l}\text { Auto-SCT vs } \\
\text { allo-SCT }\end{array}$ & 875 & $\begin{array}{l}\text { Median } 59 \text { mos } \\
\text { (among } \\
\text { survivors) }\end{array}$ & $\begin{array}{l}\text { OS } \\
\text { Auto-SCT } \\
1 \text { yr: } 90 \% ; 3 \text { yrs: } 78 \% \text {; } \\
5 \text { yrs: } 72 \% \\
\text { RIST } \\
1 \text { yr: } 80 \% ; 3 \text { yrs: } 68 \% \text {; } \\
5 \text { yrs: } 67 \% \\
\text { PFS } \\
\text { Auto-SCT } \\
1 \text { yr: } 77 \% ; 3 \text { yrs: } 57 \% \text {; } \\
5 \text { yrs: } 48 \% \\
\text { RIST } \\
1 \text { yr: } 68 \% ; 3 \text { yrs: } 62 \% ; \\
5 \text { yrs: } 57 \%\end{array}$ & NR & $\begin{array}{l}\text { Robinson et al. } \\
\text { 2013, [23] }\end{array}$ & Full text \\
\hline $\begin{array}{l}\text { France, Germany, } \\
\text { Italy, Spain, UK, } \\
\text { USA, others }\end{array}$ & $\begin{array}{l}\text { Retrospective } \\
\text { cohort }\end{array}$ & $\begin{array}{l}\text { Auto-SCT vs } \\
\text { RIC-allo-SCT }\end{array}$ & 518 & Median 61 mos & $\begin{array}{l}\text { OS } \\
\text { Allo-HCT } \\
1 \text { yr: } 77 \% ; 3 \text { yrs: } 69 \% ; \\
5 \text { yrs: } 66 \% \\
\text { Auto- } H C T \\
1 \text { yr: } 91 \% ; 3 \text { yrs: } 82 \% \text {; } \\
5 \text { yrs: } 74 \% \\
\text { PFS } \\
\text { Allo-HCT } \\
1 \text { yr: } 70 \% ; 3 \text { yrs: 61\%; } \\
5 \text { yrs: } 58 \% \\
\text { Auto- } H C T \\
1 \text { yr: } 74 \% ; 3 \text { yrs: } 51 \% ; \\
5 \text { yrs: } 41 \%\end{array}$ & NR & $\begin{array}{l}\text { Klyuchnikov } \\
\text { et al. (2015), [16] }\end{array}$ & Full text \\
\hline $\begin{array}{l}\text { France, Germany, } \\
\text { Italy, Spain, UK, } \\
\text { USA, others }\end{array}$ & $\begin{array}{l}\text { Retrospective } \\
\text { cohort }\end{array}$ & $\begin{array}{l}\text { Allo-SCT vs } \\
\text { allo-SCT }\end{array}$ & 197 & $\begin{array}{l}\text { Median } \\
\text { Allo-HCT: } 57 \text { mos } \\
\text { Auto-HCT: } 59 \\
\text { mos }\end{array}$ & $\begin{array}{l}\text { OS at } 5 \text { yrs } \\
\text { Allo-HCT: } 59 \% \\
\text { Auto-HCT: } 54 \% \\
\text { PFS at } 5 \text { yrs } \\
\text { Allo-HCT: } 51 \% \\
\text { Auto-HCT: } 36 \%\end{array}$ & NR & $\begin{array}{l}\text { Klyuchnikov } \\
\text { et al. (2016), [17] }\end{array}$ & Full text \\
\hline UK & $\begin{array}{l}\text { Retrospective } \\
\text { cohort }\end{array}$ & $\begin{array}{l}\text { BEAM-allo vs } \\
\text { BEAM-auto-SCT }\end{array}$ & 171 & $\begin{array}{l}\text { Median } \\
\text { BEAM-allo: } \\
7.7 \text { yrs } \\
\text { BEAM-auto: } \\
5.5 \text { yrs }\end{array}$ & $\begin{array}{l}\text { OS } \\
\text { BEAM-Allo } \\
1 \text { yr: } 72.5 \% ; 3 \text { yrs: } \\
68.5 \% ; 5 \text { yrs: } 66.6 \% \text {; } \\
10 \text { yrs: } 64.4 \% \\
\text { BEAM-auto } \\
1 \text { yr: } 90.1 \% ; 2 \text { yrs: } \\
78.7 \% ; 5 \text { yrs: } 62.9 \% \text {; } \\
10 \text { yrs: } 48.3 \% \\
\text { DFS } \\
\text { BEAM-allo } \\
1 \text { yr: } 63 \% ; 3 \text { yrs: } \\
57.4 \% ; 5 \text { yrs: } 53.4 \% ; \\
10 \text { yrs: } 48.1 \% \\
\text { BEAM-auto } \\
1 \text { yr: } 80.1 \% ; 2 \text { yrs: } \\
64.1 \% ; 5 \text { yrs: } 49.9 \% ; \\
10 \text { yrs: } 34.7 \%\end{array}$ & NR & $\begin{array}{l}\text { Noriega et al. } \\
\text { (2014), [33] }\end{array}$ & Full text \\
\hline
\end{tabular}

not statistically different at any of the time points examined. For the subset of patients in complete response at transplant, however, BEAM-allo seemed to show improved DFS at 10 years $(71.4$ vs $27.7 \%, \mathrm{p}=0.056)$.

One study compared outcomes among patients receiving a watch and wait strategy versus initial therapy with rituximab in low tumor-burden FL, a favorable prognostic subgroup. No difference in OS at 5 years was observed between groups (watch and wait strategy $87 \%$ vs initial therapy $88 \%$ ). Upon disease progression and receiving 
Table 4. Summary of observational studies examining clinical outcomes of treatment for follicular lymphoma (cont.).

\begin{tabular}{|c|c|c|c|c|c|c|c|c|}
\hline Country/region & Design & $\begin{array}{l}\text { Treatment } \\
\text { setting }\end{array}$ & Sample size & $\begin{array}{l}\text { Follow-up } \\
\text { (months) }\end{array}$ & $\begin{array}{l}\text { Survival/ } \\
\text { progression }\end{array}$ & Response & $\begin{array}{l}\text { Study (year), } \\
\text { Ref. }\end{array}$ & Source \\
\hline $\begin{array}{l}\text { France, Germany, } \\
\text { Italy, Spain, UK }\end{array}$ & $\begin{array}{l}\text { Retrospective } \\
\text { cohort }\end{array}$ & $\begin{array}{l}\text { RIC-allo-SCT } \\
\text { following } \\
\text { relapse after } \\
\text { auto-SCT }\end{array}$ & 183 & Median 59 mos & $\begin{array}{l}\text { OS } \\
2 \text { yrs: } 63.3 \% ; 5 \text { yrs: } \\
51.1 \% \\
\text { PFS } \\
2 \text { yrs: } 61.8 \% ; 5 \text { yrs: } \\
47.7 \%\end{array}$ & NR & $\begin{array}{l}\text { Robinson et al. } \\
\text { (2016), [22] }\end{array}$ & Full text \\
\hline Germany & $\begin{array}{l}\text { Retrospective } \\
\text { cohort }\end{array}$ & $\begin{array}{l}\text { Allo-SCT after } \\
\text { prior chemo }\end{array}$ & 146 & $\begin{array}{l}\text { Median } 9.1 \text { yrs } \\
\text { (among } \\
\text { survivors) }\end{array}$ & $\begin{array}{l}\text { OS after HCT } \\
1 \text { yr: } 67 \% ; 2 \text { yrs: } 60 \% ; \\
5 \text { yrs: } 53 \% ; 10 \text { yrs: } \\
48 \% \\
\text { EFS after HCT } \\
1 \text { yr: } 63 \% ; 2 \text { yrs: } 53 \% ; \\
5 \text { yrs: } 47 \% ; 10 \text { yrs: } \\
40 \%\end{array}$ & CR: $79 \%$ & $\begin{array}{l}\text { Heinzelmann } \\
\text { et al. (2016), [30] }\end{array}$ & Full text \\
\hline France & $\begin{array}{l}\text { Retrospective } \\
\text { cohort }\end{array}$ & $\begin{array}{l}\text { Allo-SCT after } \\
\text { response to } \\
\text { prior salvage } \\
\text { R-chemo }\end{array}$ & 59 & $\begin{array}{l}\text { Median } 37.5 \\
\text { mos }\end{array}$ & $\begin{array}{l}\text { OS at } 3 \text { yrs: } 90.5 \% \\
\text { PFS at } 3 \text { yrs: } 63.1\end{array}$ & $\begin{array}{l}\text { CR } \\
\text { Overall: } 72.9 \% \\
\text { Fludarabine: } 75 \% \\
\text { Aracytine: } 78.6 \% \\
\text { Ifosfamide: } 60 \% \\
\text { PR } \\
\text { Overall: } 16.9 \% \\
\text { Fludarabine: } 6.3 \% \\
\text { Aracytine: } 14.3 \% \\
\text { Ifosfamide: } 33.3 \%\end{array}$ & $\begin{array}{l}\text { Alcantara et al. } \\
\text { (2015), [29] }\end{array}$ & Full text \\
\hline USA & $\begin{array}{l}\text { Retrospective } \\
\text { cohort }\end{array}$ & $\begin{array}{l}\text { Long-term } \\
\text { disease control } \\
\text { after allo-SCT } \\
\text { (CIBMTR vs } \\
\text { EBMT) }\end{array}$ & 1567 & $\begin{array}{l}\text { Median } \\
\text { CIBMTR: } 58 \text { mos } \\
\text { EBMT: } 54 \text { mos }\end{array}$ & $\begin{array}{l}\text { OS at } 1 \text { yr: } \\
\text { CIBMTR: } 74 \% \text {; EBMT: } \\
75 \% \\
\text { OS at } 3 \text { yr: } \\
\text { CIBMTR: } 65 \% \text {; EBMT: } \\
67 \% \\
\text { OS at } 5 \text { yr: } \\
\text { CIBMTR: } 61 \% \text {; EBMT: } \\
62 \% \\
\text { PFS at } 1 \text { yr: } \\
\text { CIBMTR: } 66 \% \text {; EBMT: } \\
69 \% \\
\text { PFS at } 3 \text { yr: } \\
\text { CIBMTR: } 56 \% \text {; EBMT: } \\
58 \% \\
\text { PFS at } 5 \text { yr: } \\
\text { CIBMTR: } 52 \% ; \text { EBMT: } \\
52 \%\end{array}$ & NR & $\begin{array}{l}\text { Sureda et al. } \\
\text { (2015), [37] }\end{array}$ & Abstract \\
\hline NR & $\begin{array}{l}\text { Prospective } \\
\text { study }\end{array}$ & $\begin{array}{l}\text { W\&W vs active } \\
\text { therapy }\end{array}$ & 120 & Median 64 mos & $\begin{array}{l}\text { OS at } 5 \text { yrs: } 87 \% \\
\text { No difference } \\
\text { between pts initially } \\
\text { treated }(88 \%) \text { and } \\
\text { W\&W patients }(87 \% \text {; } \\
p<0.05) \\
\text { Freedom from } \\
\text { treatment failure at } \\
5 \text { yrs: } 69 \% \text { for initially } \\
\text { treated and } 79 \% \text { for } \\
\text { W\&W }(p<0.05)\end{array}$ & $\begin{array}{l}\text { CR: } 60 \% \text {, among pts } \\
\text { with progression } \\
\text { and ultimately } \\
\text { treated }\end{array}$ & $\begin{array}{l}\text { Solal-Celigny } \\
\text { et al. (2012), [36] }\end{array}$ & Full text \\
\hline Spain & $\begin{array}{l}\text { Retrospective } \\
\text { cohort }\end{array}$ & $\begin{array}{l}\text { Long-term } \\
\text { outcomes after } \\
\text { salvage } \\
\text { treatment for } \\
\text { relapse }\end{array}$ & 283 & NR & $\begin{array}{l}\text { OS at } 10 \text { yrs } \\
\text { ICT-sensitive: } 83 \% \\
\text { ICT-refractory: } 33 \% \\
\text { Death } \\
\text { ICT-sensitive: } 83 \% \\
\text { ICT-refractory: 50\% }\end{array}$ & NR & $\begin{array}{l}\text { Sorigué et al. } \\
\text { (2015), [38] }\end{array}$ & Abstract \\
\hline \multicolumn{9}{|c|}{$\begin{array}{l}\text { Auto/allo-HCT: Autologous/allogeneic hematopoietic stem cell transplantation; Auto/allo-SCT: Autologous/allogeneic stem cell transplantation; BEAM: Etoposide, Ara-C and melphalan; } \\
\text { CIBMTR: Center for International Blood and Marrow Transplant Research; CR: Complete response; DFS: Disease-free survival; EBMT: European Society for Blood and Marrow Transplan- } \\
\text { tation; EFS: Event-free survival; ICT: Immunochemotherapy; mos: Months; NR: Not reported; OS: Overall survival; PFS: Progression-free survival; PR: Partial response; pts: Patients; RIC: } \\
\text { Reduced-intensity conditioning; RIST: Reduced-intensity allo-SCT; SCT: Stem cell transplant; W\&W: Watch and wait; yrs: Years. }\end{array}$} \\
\hline
\end{tabular}




\begin{tabular}{|c|c|c|c|c|c|c|c|c|}
\hline Country/region & Design & $\begin{array}{l}\text { Treatment } \\
\text { setting }\end{array}$ & Sample size & $\begin{array}{l}\text { Follow-up } \\
\text { (months) }\end{array}$ & $\begin{array}{l}\text { Survival/ } \\
\text { progression }\end{array}$ & Response & $\begin{array}{l}\text { Study (year), } \\
\text { ref. }\end{array}$ & Source \\
\hline $\begin{array}{l}\text { France, } \\
\text { Germany, Italy, } \\
\text { Spain, UK }\end{array}$ & Phase Ib trial & $\begin{array}{l}\text { Experimental } \\
\text { chemo: obinu- } \\
\text { tuzumab }+ \text { CHOP } \\
\text { vs obinu- } \\
\text { tuzumab + FC }\end{array}$ & 56 & NR & NR & $\begin{array}{l}\text { ORR at end of } \\
\text { induction } \\
\text { CHOP: } 96 \% \\
\text { FC: } 93 \% \\
\text { CR at end of } \\
\text { induction } \\
\text { CHOP: } 39 \% \\
\text { FC: } 50 \%\end{array}$ & $\begin{array}{l}\text { Radford et al. } \\
\text { (2013), [35] }\end{array}$ & Full text \\
\hline \multirow[t]{2}{*}{$\begin{array}{l}\text { Canada, France, } \\
\text { Germany, Italy, } \\
\text { Spain, UK, USA }\end{array}$} & $\begin{array}{l}\text { Open-label, } \\
\text { Phase III study }\end{array}$ & $\begin{array}{l}\text { Experimental } \\
\text { chemo: obinu- } \\
\text { tuzumab + ben- } \\
\text { damustine } \\
\text { (G + B) }\end{array}$ & 321 & NR & NR & $\begin{array}{l}\text { CR MRD } \\
\text { positive: } 6 \% \\
\text { MRD negative: } \\
28 \% \\
\text { MRD negative: } \\
\text { G plus B } 82 \text { vs B } \\
43 \% \text {, } \\
p<0.0001 \text { ) }\end{array}$ & $\begin{array}{l}\text { Pott et al. } \\
\text { (2015), [34] }\end{array}$ & Abstract \\
\hline & Phase II trial & $\begin{array}{l}\text { 90Y-IT following } \\
\text { R-based chemo }\end{array}$ & 50 & Median 5 yrs & $\begin{array}{l}\text { Median OS: } 75.2 \\
\text { mos } \\
\text { OS at } 5 \text { yrs: } \\
77.5 \% \\
\text { Median PFS: } \\
23.1 \text { mos } \\
\text { PFS at } 1 \text { yr: } \\
94.1 \% \\
\text { PFS at } 3 \text { yrs: } \\
34.0 \% \\
\text { PFS at } 5 \text { yrs: } \\
21.9 \%\end{array}$ & $\begin{array}{l}\text { ORR } \\
\text { Week 8: } 94.2 \% \\
\text { Week 18: } 98 \% \\
\text { CR } \\
\text { Week 8: 7.7\% } \\
\text { Week 18: } 30 \%\end{array}$ & $\begin{array}{l}\text { Illidge et al. } \\
\text { (2016), [31] }\end{array}$ & Full text \\
\hline
\end{tabular}

90Y-IT: 90Y-ibritumomab-tiuxetan; Auto/allo-SCT: Autologous/allogeneic stem cell transplantation; CHOP: Cyclophosphamide, doxorubicin, vincristine and prednisone; CR: Complete response; FC: Fludarabine and cyclophosphamide; mos: Months; MRD: Minimal residual disease; NR: Not reported; ORR: Overall response rate; OS: Overall survival; PFS: Progression-free survival; wks: Weeks; yrs: Years.

active treatment, $60 \%$ of evaluable patients achieved complete response. Finally, a study by Sorigué et al. provided further evidence of the positive impact of therapy response on long-term outcomes in FL patients treated with prior first-line rituximab-based immunochemotherapy [38]. Of those patients who responded initially to primary therapy and later, upon relapse, were treated with salvage therapy, $83 \%$ were still alive at 10 years compared with only $33 \%$ of treatment-refractory patients.

\section{Clinical trials}

There were five studies reporting on R/R FL treatment trials (Table 5). Three studies examining the efficacy of experimental chemotherapy regimens were identified, with two providing evidence supporting obinutuzumab in $\mathrm{R} / \mathrm{R}$ FL [34,35]. In these two trials of obinutuzumab regimens, complete response ranged from $6 \%$ for minimal residual disease (MRD)-positive patients treated with obinutuzumab plus bendamustine [34] to $50 \%$ among patients positive for the $\mathrm{CD} 20$ antigen $\left(\mathrm{CD} 20^{+}\right)$receiving obinutuzumab + fludarabine and cyclophosphamide (FC) [35]. This Phase Ib study by Radford et al. also noted very high ORRs of $93-96 \%$ following induction with study treatments (obinutuzumab $+\mathrm{FC}$ or obinutuzumab $+\mathrm{CHOP}$ ). These results are in contrast with Ganjoo et al., who assessed ocaratuzumab in a similarly enrolled $\mathrm{CD} 20^{+} \mathrm{FL}$ population [39]]. They found ORRs around $30 \%$, 
and recommended further studies to establish a role for ocaratuzumab in CD20 $0^{+} \mathrm{FL}$. Finally, Pott et al. specifically investigated the prognostic relevance of MRD in patients treated with obinutuzumab, and found evidence suggesting that the treatment was more effective among patients with MRD-negative status versus positive (complete response rate of 28 and 6\%, respectively) [34]. The study also indicated greater depth of response with obinutuzumab plus bendamustine over bendamustine alone, with nearly twice the patients achieving MRD-negative status with obinutuzumab plus bendamustine (82 vs 43\%; $\mathrm{p}<0.0001$ ) during induction.

Illidge et al. evaluated the effectiveness of $90 \mathrm{Y}-\mathrm{IT}$ in $\mathrm{CD} 20^{+}$-relapsed patients receiving prior immunochemotherapy with rituximab [31]. After 2 months of treatment with 90Y-IT, an ORR of $98 \%$ was achieved, with $30 \%$ of the patients achieving complete response/CRu. Median OS was 75.2 months; 1- and 3-year PFS rates were 94.1 and $34.0 \%$ respectively. Only one study examined allo-SCT in patients experiencing relapse or progression after prior chemotherapy [32]. Results indicate that allo-SCT offers durable, long-term remissions for relapsed patients. The 2- and 3-year OS rates in patients who were chemosensitive undergoing RIC-allo-SCT were 84 and $82 \%$, respectively. Similarly, the 2 - and 3-year PFS rates were 73 and $71 \%$, respectively. These findings further support allo-SCT prolongation of survival and time to relapse in chemosensitive patients.

\section{Economic outcomes of treatment}

Among the studies included in this review, only one was identified that reported data on hospitalizations. No study presented evidence on medical costs or hospitalizations in R/R FL. Gyan et al. reported a hospitalization rate of $20 \%$ due to toxicity following treatment with R-NIMP among CD20 ${ }^{+}$DLBCL patients at first relapse. Patients were hospitalized for a median length of stay of 6 days [13]. The most common safety events were Grade 3-4 toxicities for neutrophils (50\%) and platelets (15\%).

\section{Discussion}

The objective of this work was to comprehensively review available data on the clinical effectiveness associated with different therapies for the treatment of R/R DLBCL and R/R FL. Although more than 2000 unique citations were identified, only 33 articles examined therapeutic effectiveness in patients with R/R DLBCL or R/R FL. Although some RCTs were included in this review, the primary objective was to identify real-world evidence; thus, not all trials were systematically identified. Furthermore, most of the RCTs included in this review were Phase I or Phase II studies, which generally have fewer enrolled patients when compared with Phase III studies for these diseases. Several of the larger RCTs that examined rituximab-based regimens in this population, including CORAL [40], the Canadian Cancer Trials Group LY.12 [41] and ORCHARRD [42], were not captured within the search algorithm due to either publication date or indexing. As a result of the design of this review, observational studies made up the majority of data included.

Sample sizes were generally higher for observational studies, which are consistent with this type of study design. A sample size of at least 50 patients was required for inclusion in the review to ensure the robustness of the data identified. While observational studies may have more potential to introduce selection bias, the majority of observational studies in this analysis sought to address this by incorporating multivariate analysis methods. Additionally, observational studies provide their own value as they demonstrate the performance of therapies used in standard clinical practice, making the results more easily generalized to the typical patient. These findings often complement and/or supplement data from clinical trials.

Overall, our review showed that in the past 5 years, evidence generation for R/R DLBCL treatment effectiveness has been limited, with studies focusing predominantly on auto- versus allo-SCT, followed by chemotherapeutic approaches, consisting of both experimental and rituximab-based combination therapies. This is not surprising, as SCT has long been considered the optimal salvage strategy following initial relapse in DLBCL (European Society for Medical Oncology Guidelines) [43,44]. While one of the highest OS rates observed (71\% at 39 months) was reported in patients treated with auto-SCT [9], there have been numerous advancements in supportive care for allo-SCT over the last decade, including improvements in donor-selection tools and RIC regimens [45-47]. These developments, along with a lack of clarity around potential deterioration of outcomes for auto-SCT in the era of immunochemotherapy, prompted Robinson et al. to conduct a study comparing different SCT treatment strategies [21]. Despite these changes, the authors observed continued greater OS and nonrelapse mortality with auto-SCT compared with allo-SCT. Within the SCT setting, longer-term survival rates were variable per study and ranged from a 3-year OS of $18 \%$ to a 4-year OS of $64 \%$. This variability is likely a result of heterogeneity within DLBCL, as the 3-year $18 \%$ OS was based on a subgroup of patients with germinal center B-cell-like DLBCL, 
whereas the 4-year $64 \%$ OS calculation was based on a population that was negative for circulating tumor disease. Response rates for SCT were reported in only one R/R DLBCL study examining allo-SCT after prior failure with auto-SCT, with an ORR of 49\% [20]. Overall, the findings of this review also indicate a high degree of variability across different DLBCL subpopulations, supporting the need for further study and use of predictive biomarkers to identify at-risk populations who may benefit from novel treatment strategies.

For DLBCL patients experiencing relapse or progression, treatment guidelines recommend high-dose chemotherapy coupled with SCT, with rituximab combination regimens commonly used for patients ineligible for SCT [44]. Over $70 \%$ of the studies included in this review examined these two treatment regimens - unsurprising as they are the dominant first-line treatments for these groups. With regard to findings pertaining to immunochemotherapy in $\mathrm{R} / \mathrm{R}$ DLBCL, the prognosis among patients receiving rituximab-based regimens was inferior to SCT, with median OS statistics ranging from 10.8 to 28.4 months. Not surprisingly, the lowest OS and PFS outcomes were observed in studies that exclusively focused on patients ineligible for auto-SCT due to age or co-morbidity [7,19]. The median age range (64-76 years) also tended to be older for studies examining rituximab-based regimens, with a higher proportion of patients having more advanced disease $(61-76 \%$ stage 3 or 4$)$ relative to studies in the SCT setting. Five-year OS rates for BEAM conditioning regimens (69-86\%) were among the highest of all the studies included in this review, with one study evaluating different conditioning regimens showing a slight 5-year OS advantage (but no difference in DFS) for Z-BEAM over BEAM/R. BEAM response rates were not frequently reported, but the highest response rate (84\%) was observed in a Phase II study evaluating BEAM conditioning regimen.

Among the RCTs included in this study, most were focused on survival outcomes for experimental chemotherapeutic regimens. The highest OS reported among these studies was a median of 28.4 months for a rituximab-based regimen. This particular study also reported the highest median PFS of 10.5 months. While these median survival times are higher than what was reported for observational studies, this may have been due to age or performance status differences between the study populations. Six of the included clinical trials reported response outcomes, with the highest being an ORR of $96 \%$ in a study of vorinostat, gemcitabine and busulfan/melphalan [18].

A recently published patient-level analysis [48] pooled the results of two pivotal rituximab trials (CORAL and CCTG LY.12) with two large observational cohorts to examine response rates and survival for patients with refractory DLBCL. Pooled results showed a 26\% ORR with a range of $20-31 \%$ among the four included studies. Findings for pooled OS were similar: median OS of 6.3 months (range of 5.9-7.0 months among included studies; $20 \%$ of patients alive at 2 years). The results of this retrospective analysis are significant for several reasons: the authors concluded that, overall, there are limited data published for this patient population, which is confirmatory to the findings of the current review. Even with the current treatment landscape including multiple rituximab-based therapies, response and survival outcomes for refractory DLBCL remain poor. The rate of response and median survival was similar across study designs.

Similar to R/R DLBCL, there were limited data on treatment-related effectiveness outcomes reported for $R / R$ $\mathrm{FL}$ in the past 5 years. Consistent with its more indolent pathophysiology, survival outcomes for R/R FL were generally better than those reported for R/R DLBCL.

Approximately half of studies focusing on R/R FL examined outcomes for auto-SCT or allo-SCT. This was surprising as treatment guidelines suggest reserving SCT for only select cases [44]. Five-year OS with SCT ranged from 51 to $72 \%$. There was some evidence indicating that survival is improved with auto-SCT versus RIC-alloSCT, although this may have been attributable to differences in disease stage at diagnosis and was not consistently observed in the available literature. As with R/R DLBCL, data on treatment response rates in the SCT setting were limited in observational studies, more prevalent among RCTs, but were overall quite variable across studies and patient subgroups.

According to treatment guidelines, chemotherapy regimens containing rituximab should be the mainstay of treatment for R/R FL [43], as NCCN guidelines currently recommend SCT only for patients who progress within the first 6 months of first-line maintenance with rituximab, or for patients on their second or third remission. When examining studies focused on chemotherapy regimens, the highest ORR (96\%) was reported in a Phase IB trial for patients receiving obinutuzumab in combination with CHOP [35]. ORR ranged from 30-90\%, and complete response ranged from $6 \%$ for MRD-positive patients receiving obinutuzumab to $50 \%$ for $\mathrm{CD} 20^{+}$patients receiving obinutuzumab with FC. In contrast, the data for the experimental agent ocaratuzumab were not as robust, with an ORR of only around $30 \%$ in patients with $\mathrm{R} / \mathrm{R} \mathrm{CD} 20^{+} \mathrm{FL}$. As with DLBCL, patient populations of studies focusing on the SCT setting were generally younger compared with those treated with chemotherapy. 
As with all systematic literature reviews, there are certainly limitations in the scope of what the review can address. The objective of this SLR was to identify studies of patients with R/R DLBCL or R/R FL, although the search allowed for inclusion of studies including all DLBCL or FL patients. Patient demographics, such as age, were different among patients who received stem cell therapies and those who received other treatments, making it inherently difficult to truly assess the effectiveness of each treatment on clinical outcomes. As discussed earlier, in studies included for R/R FL, SCT was the most prevalent therapy assessed, which is in contrast to what guidelines would suggest. Given the higher reporting of survival outcomes observed in the studies evaluating SCT, it is possible that some bias toward these studies may have been introduced by the search strategy's focus on effectiveness outcomes. Finally, this review did not address newer therapies, including chimeric antigen receptor T-cell therapies, gene treatments for R/R DLBCL. Axicabtagene ciloleucel gained the US FDA approval in October of 2017 for large B-cell lymphoma (including DLBCL) after failure with at least two other therapy types; however, studies examining this treatment were not published until after the review's timeframe. Similarly, tisagenlecleucel gained approval from the FDA in early May 2018 for the treatment of R/R DLBCL patients. Both of these treatments could have significant impact on the treatment landscape. Axicabtagene ciloleucel showed a complete response rate of $54 \%$ in a multinational clinical trial [49] and tisagenlecleucel treatment showed an ORR of 50\% (32\% complete response) in a pivotal Phase II trial [50].

\section{Conclusion}

Overall, the results presented here indicate that there are limited data regarding real-world effectiveness and economic outcomes for approved treatment strategies and clinical outcomes in patients with R/R DLBCL or R/R FL. Among studies analyzed for R/R DLBCL, treatment courses ranged from SCT to combination chemotherapy, although most of the studies examined SCT. Survival rates and treatment responses were variable and survival outcomes were more likely to be reported in studies that examined SCT as a treatment modality, whereas studies examining experimental regimens were more likely to report response outcomes. Among studies analyzed for R/R FL, treatment settings ranged from SCT to chemotherapy, including experimental types and 90Y-IT. Survival outcomes were more often reported in studies evaluating SCT, while information on response was limited in these SCT studies, and survival rates were variable. Response rates in patients who received experimental chemotherapy were also variable. This variability in survival and response rates is likely a consequence of heterogeneity within DLBCL and FL populations, and suggests a need for further research on prognostic biomarkers to guide therapy choice in R/R DLBCL and FL patients. For both DLBCL and FL, SCT-based treatment strategies were the most analyzed in this review. However, SCT can be used in only a subset of patients, as it is not currently recommended for the elderly or those with multiple comorbidities. These findings suggest that treatment options for patients ineligible for SCT remain a significant unmet need.

\section{Future perspective}

Many of the current studies of DLBCL and FL included in this review examined SCT. Indeed, the studies suggest that patients treated with this strategy have the best prognosis in terms of both OS and overall response. However, SCT can be used in only a subset of patients, as it is not currently recommended for the elderly or those with multiple co-morbidities. A lack of viable treatment options with proven long-term results for these patients currently represents a significant gap in care. Further research into nontransplant options is likely to gain momentum in order to fill this critical unmet need, along with a push to identify predictive biomarkers to distinguish the patients who are most likely to benefit from these novel treatment strategies. Additionally, given the positive long-term results associated with SCT, we are likely to see more prognostic studies further delineating the specific subsets of patients who are the best candidates for this therapy. Lastly, RIC regimens for allo-SCT have already reduced the high treatment-related mortality historically associated with this procedure, so we expect additional research into these regimens in an effort to expand the numbers of patients with R/R DLBCL and R/R FL who can benefit from SCT.

\section{Acknowledgements}

The authors acknowledge Melanie Jardim for medical writing, and Janet Dooley for editorial support in the preparation of this manuscript. These individuals are salaried employees of Evidera. 
- Non-Hodgkin lymphoma (NHL) is the most prevalent hematological malignancy. Diffuse large B-cell lymphoma (DLBCL) and follicular lymphoma (FL) are the most common types of NHL, accounting for approximately $65 \%$ of all NHLs.

- A frequently used regimen for both indications is rituximab given in combination with the regimen of cyclophosphamide, doxorubicin, vincristine and prednisone (R-CHOP).

- R-CHOP significantly improves survival in FL and DLBCL.

- Approximately a third of DLBCL patients develop relapsed or refractory $(\mathrm{R} / \mathrm{R})$ disease. Although $\mathrm{FL}$ is a more indolent form of $\mathrm{NHL}$, there is currently no cure and relapses occur.

- Current therapeutic options for patients include stem cell transplant (SCT) and chemotherapy/immunotherapy. More effective therapies for patients ineligible for SCT remain an important objective.

- This review systematically evaluated the evidentiary base for therapies used in R/R DLBCL or R/R FL.

- There was limited evidence on effectiveness outcomes for R/R DLBCL and R/R FL in the past 5 years. Treatments included SCT and chemotherapy. No study of R/R FL examined rituximab-based chemotherapy, despite treatment guidelines describing it as a treatment mainstay.

- The majority of observational studies included for R/R DLBCL examined the effectiveness of either autologous (auto-) SCT or allogeneic (allo-) SCT. One of the highest overall survival (OS) rates observed (71\% at 39 months) was reported in patients treated with auto-SCT; this mode of therapy is considered the optimal salvage strategy following initial relapse.

- For DLBCL, the prognosis among those receiving rituximab-based regimens was inferior to SCT, with median OS from 10.8 to 28.4 months. The lowest OS and progression-free survival were observed in studies that exclusively focused on patients ineligible for auto-SCT due to age or co-morbidity.

- High-dose chemotherapy followed by auto-SCT is a well-defined treatment modality for R/R DLBCL. Five-year OS rates for etoposide, Ara-C and melphalan (BEAM) conditioning regimens ranged from 69 to $86 \%$ and were among the highest reported in the review.

- Approximately half of FL studies examined outcomes for auto-SCT or allo-SCT, which was surprising as treatment guidelines suggest reserving SCT for only select cases. 5-year OS ranged from 51 to $72 \%$. Some evidence indicates that survival is improved with auto-SCT versus reduced-intensity conditioning-allo-SCT, although this may have been attributable to differences in baseline disease stage and was not consistently observed in the available literature.

- Experimental chemotherapy regimens were examined in several studies of FL. The highest overall response rate $(96 \%)$ was reported in a Phase IB trial examining obinutuzumab in combination with CHOP. In contrast, data for the experimental agent ocaratuzumab reported an overall response rate of only $30 \%$ in $\mathrm{CD}_{20} 0^{+}$patients.

Financial \& competing interests disclosure

Millennium Pharmaceuticals, Inc., a wholly owned subsidiary of Takeda Pharmaceutical Company Limited, funded this project and article. They provided funding to Evidera to provide research, technical support and writing assistance. The authors R Huelin, M Stokes, Y Guo, M Hoog and T Bhagnani are all salaried employees of Evidera, a research and consulting firm for the biopharma industry, and are prohibited from accepting any remuneration or honoraria directly from Evidera's clients. The authors A Galaznik, J Bell and Y Shou are all salaried employees and stock holders in Millennium Pharmaceuticals, Inc., a wholly owned subsidiary of Takeda Pharmaceutical Company Limited. The authors have no other relevant affiliations or financial involvement with any organization or entity with a financial interest in or financial conflict with the subject matter or materials discussed in the manuscript apart from those disclosed.

Writing assistance was utilized in the production of this manuscript.

\section{Authors' contributions}

A Galaznik and R Huelin were responsible for conception and design of the review; analysis and interpretation of the evidence; revision, review and approval of manuscript and confirmation of accuracy. M Stokes, Y Guo, M Hoog, T Bhagnani, J Bell and Y Shou were responsible for analysis and interpretation of the evidence; manuscript drafts and revisions; approval of the manuscript and confirmation of accuracy.

Open access

This work is licensed under the Creative Commons Attribution 4.0 License. To view a copy of this license, visit http://creativecommons.org/licenses/by/4.0/ 


\section{References}

Papers of special note have been highlighted as: $\bullet$ of interest; $\bullet \bullet$ of considerable interest

1. Armitage JO, Gascoyne RD, Lunning MA, Cavalli F. Non-Hodgkin lymphoma. Lancet 390(10091), 298-310 (2017).

2. Nowakowski GS, Chiappella A, Witzig TE et al. ROBUST: lenalidomide-R-CHOP versus placebo-R-CHOP in previously untreated ABC-type diffuse large B-cell lymphoma. Future Oncol. 12(13), 1553-1563 (2016).

3. National Comprehensive Cancer Network. NCCN Clinical Practice Guidelines in Oncology (NCCN Guidelines ${ }^{\circledR}$ ) B-cell Lymphomas, version 7.2017. (2017). www.nccn.org/professionals/physician_gls/pdf/b-cell.pdf

4. Zhou Z, Sehn LH, Rademaker AW et al. An enhanced International Prognostic Index (NCCN-IPI) for patients with diffuse large B-cell lymphoma treated in the rituximab era. Blood 123(6), 837-842 (2014).

5. $\quad$ PDQ ${ }^{\circledR}$ Adult Treatment Editorial Board. Adult non-Hodgkin lymphoma treatment (PDQ ${ }^{\circledR}$ )-health professional version. (2017). www.cancer.gov/types/lymphoma/hp/adult-nhl-treatment-pdq

6. Amin AD, Peters TL, Li L et al. Diffuse large B-cell lymphoma: can genomics improve treatment options for a curable cancer? Cold Spring Harb. Mol. Case Stud. 3(3), a001719 (2017).

7. Arcari A, Chiappella A, Spina M et al. Safety and efficacy of rituximab plus bendamustine in relapsed or refractory diffuse large B-cell lymphoma patients: an Italian retrospective multicenter study. Leuk. Lymphoma. 57(8), 1823-1830 (2015)

8. Avivi I, Canals C, Vernant JP et al. Matched unrelated donor allogeneic transplantation provides comparable long-term outcome to HLA-identical sibling transplantation in relapsed diffuse large B-cell lymphoma. Bone Marrow Transplant. 49(5), 671-678 (2014).

9. Avivi I, Boumendil A, Finel HH et al. Autologous stem cell transplantation for primary mediastinal B cell lymphoma in the rituximab era: a retrospective study by the EBMT Lymphoma Working Party. American Society of Hematology (ASH) 56 th Annual Meeting and Exposition. San Francisco, CA, USA, 6-9 December 2014 (Abstract 1195).

- Showed the highest overall survival.

10. Farooq F, Maurer MJ, Ansell SM et al. Treatment patterns and outcomes of DLBCL after failure of front-line immunochemotherapy. American Society of Hematology (ASH) 57th Annual Meeting and Exposition. Orlando, FL, USA, 5-8 December 2015 (Abstract 2683).

11. Fenske TS, Ahn KW, Graff TM et al. Allogeneic transplantation provides durable remission in a subset of DLBCL patients relapsing after autologous transplantation. Br. J. Haematol. 174(2), 235-248 (2016).

12. Flinn IW, van der Jagt R, Kahl BS et al. Randomized trial of bendamustine-rituximab or R-CHOP/R-CVP in first-line treatment of indolent NHL or MCL: the BRIGHT study. Blood 123(19), 2944-2952 (2014)

13. Gyan E, Damotte D, Courby $\mathrm{S}$ et al. High response rate and acceptable toxicity of a combination of rituximab, vinorelbine, ifosfamide mitoxantrone and prednisone for the treatment of diffuse large B-cell lymphoma in first relapse: results of the R-NIMP GOELAMS study. Br. J. Haematol. 162(2), 240-249 (2013).

-. Reports the limited economic data on hospitalizations that we could find.

14. Herrera AF, Mei MG, Low L et al. Double expressing (MYC/BCL2) and double-hit diffuse large B-cell lymphomas have inferior survival following autologous stem cell transplantation. Presented at: American Society of Hematology (ASH) 57th Annual Meeting and Exposition Orlando, FL, USA, 5-8 December 2015 (Abstract 522)

15. Khouri I, Sui D, Turturro F et al. In-vivo purging with rituximab (R) followed by Z/BEAM vs BEAM/R autologous stem cell conditioning for relapsed diffuse large B-cell lymphoma (DLBCL) patients (pts): mature results from a combined analysis of 3 trials. Presented at: American Society of Hematology (ASH) 57th Annual Meeting and Exposition. Orlando, FL, USA, 5-8 December 2015 (Abstract 3192).

16. Klyuchnikov E, Bacher U, Kröger NM et al. Reduced-intensity allografting as first transplantation approach in relapsed/refractory Grades one and two follicular lymphoma provides improved outcomes in long-term survivors. Biol. Blood Marrow Transplant. 21(12), 2091-2099 (2015)

17. Klyuchnikov E, Bacher U, Woo Ahn K et al. Long-term survival outcomes of reduced-intensity allogeneic or autologous transplantation in relapsed Grade 3 follicular lymphoma. Bone Marrow Transplant. 51(1), 58-66 (2016).

18. Nieto Y, Valdez BC, Thall PF et al. Vorinostat combined with high-dose gemcitabine, busulfan, and melphalan with autologous stem cell transplantation in patients with refractory lymphomas. Biol. Blood Marrow Transplant. 21(11), 1914-1920 (2015).

19. Ohmachi K, Niitsu N, Uchida T et al. Multicenter Phase II study of bendamustine plus rituximab in patients with relapsed or refractory diffuse large B-cell lymphoma. J. Clin. Oncol. 31(17), 2103-2109 (2013).

20. Rigacci L, Puccini B, Dodero A et al. Allogeneic hematopoietic stem cell transplantation in patients with diffuse large B cell lymphoma relapsed after autologous stem cell transplantation: a GITMO study. Ann. Hematol. 91(6), 931-939 (2012).

21. Robinson SP, Boumendil A, Finel $\mathrm{H}$ et al. Autologous stem cell transplantation for relapsed/refractory diffuse large B-cell lymphoma: efficacy in the rituximab era and comparison to first allogeneic transplants. A report from the EBMT Lymphoma Working Party. Bone Marrow Transplant. 51(3), 365-371 (2016).

-• Compares allogeneic versus autologous-stem cell transplantation (allo-/auto-SCT). 
22. Robinson SP, Boumendil A, Finel $\mathrm{H}$ et al. Reduced intensity allogeneic stem cell transplantation for follicular lymphoma relapsing after an autologous transplant achieves durable long term disease control. An analysis from the Lymphoma Working Party of the EBMT. Ann. Oncol. 27(6), 1088-1094 (2016).

23. Robinson SP, Canals C, Luang JJ et al. The outcome of reduced intensity allogeneic stem cell transplantation and autologous stem cell transplantation when performed as a first transplant strategy in relapsed follicular lymphoma: an analysis from the Lymphoma Working Party of the EBMT. Bone Marrow Transplant. 48(11), 1409-1414 (2013).

•. Compares allo-SCT with reduced-intensity conditioning-auto-SCT.

24. Rovira J, Valera A, Colomo L et al. Prognosis of patients with diffuse large B cell lymphoma not reaching complete response or relapsing after frontline chemotherapy or immunochemotherapy. Ann. Hematol. 94(5), 803-812 (2015).

-. Examines longer term survival (5 years).

25. Lisenko K, McClanahan F, Schoning T et al. Minimal renal toxicity after Rituximab DHAP with a modified cisplatin application scheme in patients with relapsed or refractory diffuse large B-cell lymphoma. BMC Cancer 16, 267 (2016).

26. Khouri I, Saliba R, Xu-Monette Z et al. Outcomes following allogeneic stem cell transplantation (alloSCT) in patients with primary mediastinal (PMBL), germinal center B (GCB) and non-GCB cell-like diffuse large B cell lymphomas (DLBCL). Presented at: American Society of Hematology (ASH) 56th Annual Meeting and Exposition, 6-9 December 2014, San Francisco, CA. Blood 124(21), Abstract 2563 (2014).

27. Czucman MS, Davis A, Linton KM et al. Phase $2 / 3$ multicenter, randomized study comparing the efficacy and safety of lenalidomide versus investigator's choice in relapsed/refractory DLBCL. Presented at: American Society of Hematology (ASH) 56th Annual Meeting and Exposition. San Francisco, CA, USA, 6-9 December 2014 (Abstract 628).

28. Isidori A, Guidi S, Scalzulli PR et al. Benda-beam high-dose therapy prior to auto-SCT is effective in resistant/relapsed DLBCL. Presented at: American Society of Hematology (ASH) 57th Annual Meeting and Exposition. Orlando, FL, USA, 5-8 December 2015 (Abstract 1999).

29. Alcantara M, Dupuis J, Mareschal S et al. PET/CT before autologous stem cell transplantation predicts outcome in refractory/relapsed follicular lymphoma. Eur. J. Nucl. Med. Mol. Imaging 42(2), 215-221 (2015).

- Examines 3-year survival and shows importance of prognostic factors.

30. Heinzelmann F, Bethge W, Beelen DW et al. Allogeneic hematopoietic cell transplantation as curative therapy for non-transformed follicular lymphomas. Bone Marrow Transplant. 51(5), 654-662 (2016).

31. Illidge TM, McKenzie HS, Mayes $S$ et al. Short duration immunochemotherapy followed by radioimmunotherapy consolidation is effective and well tolerated in relapsed follicular lymphoma: 5-year results from a UK National Cancer Research Institute Lymphoma Group study. Br. J. Haematol. 173(2), 274-282 (2016).

32. Laport GG, Wu J, Logan B et al. Reduced intensity conditioning with fludarabine, cyclophosphamide, and high dose rituxan for allogeneic hematopoietic cell transplantation for follicular lymphoma: a Phase II multicenter trial from the Blood and Marrow Transplant Network (BMT CTN). Biol. Blood Marrow Transplant. doi:10.1016/j.bbmt.2016.04.014 (2016) (Epub ahead of print).

33. Noriega $\mathrm{V}$, Kaur H, Devereux $\mathrm{S}$ et al. Long term follow-up of BEAM-autologous and BEAM-alemtuzumab allogeneic stem cell transplantation in relapsed advanced stage follicular lymphoma. Leuk. Res. 38(7), 737-743 (2014).

34. Pott C, Belada D, Danesi N et al. Analysis of minimal residual disease in follicular lymphoma patients in gadolin, a Phase III study of obinutuzumab plus bendamustine versus bendamustine in relapsed/refractory indolent non-Hodgkin lymphoma. Presented at: American Society of Hematology (ASH) 57th Annual Meeting and Exposition. Orlando, FL, USA, 5-8 December 2015 (Abstract 3978).

35. Radford J, Davies A, Cartron G et al. Obinutuzumab (GA101) plus CHOP or FC in relapsed/refractory follicular lymphoma: results of the GAUDI study (BO21000). Blood 122(7), 1137-1143 (2013).

36. Solal-Céligny P, Bellei M, Marcheselli L et al. Watchful waiting in low-tumor burden follicular lymphoma in the rituximab era: results of an F2-study database. J. Clin. Oncol. 30(31), 3848-3853 (2012).

37. Sureda A, Zhang MJ, Dreger P et al. Allogeneic stem cell transplantation for relapsed / refractory (R/R) follicular lymphoma (FL). A joint study between the European Society for Blood and Marrow Transplantation (EBMT) and the Center for International Blood and Marrow Transplant Research (CIBMTR). Presented at: American Society of Hematology (ASH) 57th Annual Meeting and Exposition. Orlando, FL, USA, 5-8 December 2015 (Abstract 198).

38. Sorigué M, Sancho JM, Mercadal S et al. Prevalence, predictive factors therapy and outcome of patients with follicular lymphoma refractory to first line immunochemotherapy. Presented at: American Society of Hematology (ASH) 57th Annual Meeting and Exposition. Orlando, FL, USA, 5-8 December 2015 (Abstract 1510).

- Examines longer term outcomes in follicular lymphoma for patients treated with rituximab.

39. Ganjoo KN, de Vos S, Pohlman BL et al. Phase $1 / 2$ study of ocaratuzumab, an Fc-engineered humanized anti-CD20 monoclonal antibody, in low-affinity Fc-gamma-RIIIa patients with previously treated follicular lymphoma. Leuk. Lymphoma 56(1), 42-48 (2015).

40. Gisselbrecht C, Glass B, Mounier N et al. Salvage regimens with autologous transplantation for relapsed large B-cell lymphoma in the rituximab era. J. Clin. Oncol. 28(27), 4184-4190 (2010). 
41. Crump M, Kuruvilla J, Couban S et al. Randomized comparison of gemcitabine, dexamethasone, and cisplatin versus dexamethasone, cytarabine, and cisplatin chemotherapy before autologous stem-cell transplantation for relapsed and refractory aggressive lymphomas: NCIC-CTG LY.12. J. Clin. Oncol. 32(31), 3490-3496 (2014).

42. van Imhoff GW, McMillan A, Matasar MJ et al. Ofatumumab versus Rituximab salvage chemoimmunotherapy in relapsed or refractory diffuse large B-cell lymphoma: the ORCHARRD study. J. Clin. Oncol. 35(5), 544-551 (2017).

43. Dreyling M, Ghielmini M, Rule $S$ et al. Newly diagnosed and relapsed follicular lymphoma: ESMO Clinical Practice Guidelines for diagnosis, treatment and follow-up. Ann. Oncol. 27(Suppl. 5), v83-v90 (2016).

44. Tilly H, Gomes da Silva M, Vitolo U et al. Diffuse large B-cell lymphoma (DLBCL): ESMO Clinical Practice Guidelines for diagnosis, treatment and follow-up. Ann. Oncol. 26(Suppl. 5), v116-125 (2015).

45. Rezvani AR, Storer B, Maris M et al. Nonmyeloablative allogeneic hematopoietic cell transplantation in relapsed, refractory, and transformed indolent non-Hodgkin's lymphoma. J. Clin. Oncol. 26(2), 211-217 (2008).

46. Sirvent A, Dhedin N, Michallet $\mathrm{M}$ et al. Low nonrelapse mortality and prolonged long-term survival after reduced-intensity allogeneic stem cell transplantation for relapsed or refractory diffuse large B cell lymphoma: report of the Societe Francaise de Greffe de Moelle et de Therapie Cellulaire. Biol. Blood Marrow Transplant. 16(1), 78-85 (2010).

47. Thomson KJ, Morris EC, Bloor A et al. Favorable long-term survival after reduced-intensity allogeneic transplantation for multiple-relapse aggressive non-Hodgkin's lymphoma. J. Clin. Oncol. 27(3), 426-432 (2009).

48. Crump M, Neelapu SS, Farooq U et al. Outcomes in refractory diffuse large B-cell lymphoma: results from the international SCHOLAR-1 study. Blood 130(16), 1800-1808 (2017).

49. Neelapu SS, Locke FL, Bartlett NL et al. Axicabtagene ciloleucel CAR T-cell therapy in refractory large B-cell lymphoma. N. Engl. J. Med. 377(26), 2531-2544 (2017).

50. Schuster SJ, Bishop MR, Tam CS et al. Primary analysis of Juliet: a global, pivotal, Phase 2 trial of CTL019 in adult patients with relapsed or refractory diffuse large B-cell lymphoma. Presented at: American Society of Hematology (ASH) 59th Annual Meeting and Exposition. Atlanta, GA, USA, 9-12 December 2017 (Abstract 577). 